\title{
Precision in Liver Surgery
}

\author{
jiahong Dong, MD, PhD, FACS ${ }^{1}$ ShiZhong Yang, MD, $\mathrm{PhD}^{1} \quad$ jianping Zeng, $\mathrm{MD}, \mathrm{PhD}^{1}$ \\ Shouwang Cai, MD, PhD ${ }^{1}$ Wenbin Ji, MD ${ }^{1}$ Weidong Duan, MD, $\mathrm{PhD}^{1}$ Aiqun Zhang, MD, PhD ${ }^{1}$ \\ Weizheng Ren, MD ${ }^{1} \quad$ Yinzhe Xu, MD ${ }^{1} \quad$ jingwang Tan, MD, $\mathrm{PhD}^{1} \quad$ Xiangyang Bu, MD ${ }^{1} \quad$ Ning Zhang, MD ${ }^{1}$ \\ Xuedong Wang, $\mathrm{MD}^{1} \quad$ Xianqiang Wang, $\mathrm{MD}^{1} \quad$ Xiangfei Meng, $\mathrm{MD}^{1} \quad$ Kai Jiang, $\mathrm{MD}, \mathrm{PhD}^{1}$ \\ Wanqing Gu, MD ${ }^{1}$ Zhiqiang Huang, MD, $\operatorname{FRCSEd}(H)^{1}$
}

\author{
${ }^{1}$ Hospital and Institute of Hepatobiliary Surgery, Chinese PLA General \\ Hospital, Beijing, China \\ Address for correspondence Jiahong Dong, MD, PhD, Hospital and \\ Institute of Hepatobiliary Surgery, Chinese PLA General Hospital, 28 \\ Fuxing Road, Haidian District, Beijing, 100853, China \\ Semin Liver Dis 2013;33:189-203. \\ (e-mail: Dongjh301@163.com).
}

\begin{abstract}
Keywords

- precision surgery

- liver surgery

- certainty

- multiobjective optimization

Continuous theoretical and technological progress in the face of increasing expectations for quality health care has transformed the surgical paradigm. The authors systematically review these historical trends and propose the novel paradigm of "precision surgery," featuring certainty-based practice to ensure the best result for each patient with multiobjective optimization of therapeutic effectiveness, surgical safety, and minimal invasiveness. The main characteristics of precision surgery may be summarized as determinacy, predictability, controllability, integration, standardization, and individualization. The strategy of precision in liver surgery is to seek a balance of maximizing the removal of the target lesion, while maximizing the functional liver remnant and minimizing surgical invasiveness. In this article, the authors demonstrate the application of precision approaches in specific settings in complex liver surgery. They propose that the concept of precision surgery should be considered for wider application in liver surgery and other fields as a step toward the ultimate goal of perfect surgery.
\end{abstract}

Surgery has passed through an intuitive and an empirical era and has now entered its modern phase characterized by everincreasing certainty in surgical practice. ${ }^{1}$ The enormous progress in biomedicine, the rise of evidence-based medicine, and the consensus on the need for humanistic patient care in the $21^{\text {st }}$ century have laid a foundation for a new surgical paradigm. This surgical paradigm featuring high-certainty clinical practice would enable simultaneous optimization of therapeutic effectiveness, surgical safety, and minimal invasiveness. We argued for the necessity of a paradigm shift in liver surgery, and advocated "precision liver resection" as a surgical concept for the first time in 2006,,3 and later extended the concept of "precision liver resection" to "precision liver surgery." ${ }^{4,5}$ This concept is widely applicable within various surgical fields and is embraced by both the Chinese and international community. In this review, we advocate the new paradigm of "precision surgery" and attempt to establish its theoretical and technological framework by examining the evolution of surgery, the advances in surgical science and technology, and the health care needs within modern society. "Precision" does not just refer to the accuracy of operative manipulation, some idealized procedure, or a particular advanced technology, 6,7 "Precision" as we define it is a new approach to surgery and its derived system of theories and technologies covering all the elements of surgical practice, including preoperative evaluation, clinical decision making, surgical planning, operative manipulation, and perioperative management. $^{3}$

\section{Emergence of Precision Surgery}

Surgery, as a direct and profound exploration of the human body, has always been a symphony of science and art. In this everlasting symphony, the surgical paradigm has evolved
Issue Theme Liver Surgery in 2013; Guest Editors, Jean C. Emond, MD, and Michael D. Kluger, MD, MPH
Copyright (c) 2013 by Thieme Medical Publishers, Inc., 333 Seventh Avenue, New York, NY 10001, USA. Tel: +1(212) 584-4662.
DOI http://dx.doi.org/ 10.1055/s-0033-1351781. ISSN $0272-8087$. 
from intuitive to empirical during the past century. The concept of paradigm, proposed by Thomas Kuhn, refers to the set of practices that define a scientific discipline. ${ }^{8}$ In surgery, the paradigm consists of professional knowledge, techniques and experiences, practical principles, strategies, and objectives, which constitute a framework for the delivery of surgical service.

Surgery originally emerged as an intuitive practice with a low standard of safety at the outset. The establishment of anesthesia, sterilization, and transfusion in the $19^{\text {th }}$ century provided a basic promise of surgical safety, which granted surgery a vast potential to innovate and pursue increasing therapeutic effectiveness. ${ }^{1}$ Since the beginning of the $20^{\text {th }}$ century, the accumulation of surgical experience and the development of preclinical medicine gradually transformed surgery into a science of intervening into the pathologic course of disease and restoring functional integrity of organs. From then on, a systematic paradigm came into being, to which we now refer as empirical surgery. ${ }^{1,9}$ Since the early 1960 s, the stunning progress in the life sciences and information technology has taken surgery into a golden era. Together with the accumulation of experience accelerated by surgical specialization, the capability of surgical intervention has been greatly expanded. Various aggressive procedures for lesion removal, functional restoration, and organ replacement were established, which allowed more patients to undergo a radical procedure rather than palliative care. ${ }^{10-15}$ In parallel, the rise of minimally invasive surgery in the past 30 years permits the performance of procedures endoscopically and laparoscopically, avoiding open surgical access and decreasing invasiveness. $^{16-21}$

Therapeutic effectiveness, surgical safety, and minimal invasiveness are the principal objectives (which may be referred to as "30") of surgery. The simultaneous optimization of the 30 , or multiobjective optimization, is the premise of best surgical outcomes. Despite the significant improvement in the capacity and capability of surgery in each objective over time, the status quo remains far from fully satisfactory, especially in some challenging areas such as liver surgery. Because most liver surgery is performed in the treatment of localized lesions, to achieve the objective of therapeutic effectiveness we must maximize lesion removal. At the same time, we attempt to promote surgical safety via maximizing the functional liver remnant, and above all, we strive to reduce concomitant surgical trauma by minimizing surgical invasiveness. These three elements (which may be referred to as "3M") of contemporary surgical practice at times mutually conflict, and resolution of this balance is a prerequisite of multiobjective optimization and would ensure the best surgical outcomes.

The primary limit to identifying this resolution lies in the level of uncertainty throughout the surgical process, such as insufficient understanding of the biology of the disease in the individual patient, limitations of imaging technology for both staging and surgical planning, inaccuracy in functional assessment of organs, the intrinsic limitations of surgical techniques, and anesthesia. Facing these uncertainties, surgeons would attempt to offset the undesired consequences with experience. The experienced surgeon might be capable of overcoming these limitations sometimes, but unfortunately, experience-based practice is not reliably reproducible because intuition and unsystematic clinical experience are individually variable and far from sufficient for sound clinical decision making. ${ }^{22}$ On this condition, accurate control in a single objective would be difficult enough, leaving multiobjective optimization an unattainable dream. It is impossible to break this impasse, unless a sufficient degree of certainty is achieved throughout surgical process, the risk of the residual uncertainty is managed with scientific decision making, and a balanced surgical strategy is formulated. Obviously, a paradigm shift in the field of surgery is then called for (-Fig. 1).

In summary, with the enormous progress in biomedicine, the rise of evidence-based medicine, ${ }^{23,24}$ and the consensus on the need for humanistic patient care $\mathrm{e}^{25}$ in the $21^{\text {st }}$ century, a new surgical paradigm must establish a certainty-based practice with a strategy of multiobjective optimization. This paradigm has evolved from conventional surgery and has been modernized through integration with state-of-the-art science and technology. It will provide a framework to precisely balance maximal lesion removal and organ sparing, with minimal surgical invasiveness (3M). In this way, a quality service of surgery with multiobjective optimization accommodating therapeutic effectiveness, surgical safety, and minimal invasiveness (30) can be offered to ensure maximized benefit and recovery for each patient. The process of care can be analyzed according to the elements of practice, creating a continuously reinforcing cycle of surgical quality. In addition, surgical training may be structured around these elements to diminish the impact of individual variability and increase the reliability of surgical care. We have chosen the term "precision surgery" for this paradigm (-Fig. 2).

\section{Characteristics of Precision Surgery}

The fundamental philosophy that underlies precision surgery is scientific determinism, the belief that each event can be tracked definitively to its cause. ${ }^{26}$ In medical practice based on determinism, absolute certainty in assessment, diagnosis, and treat-

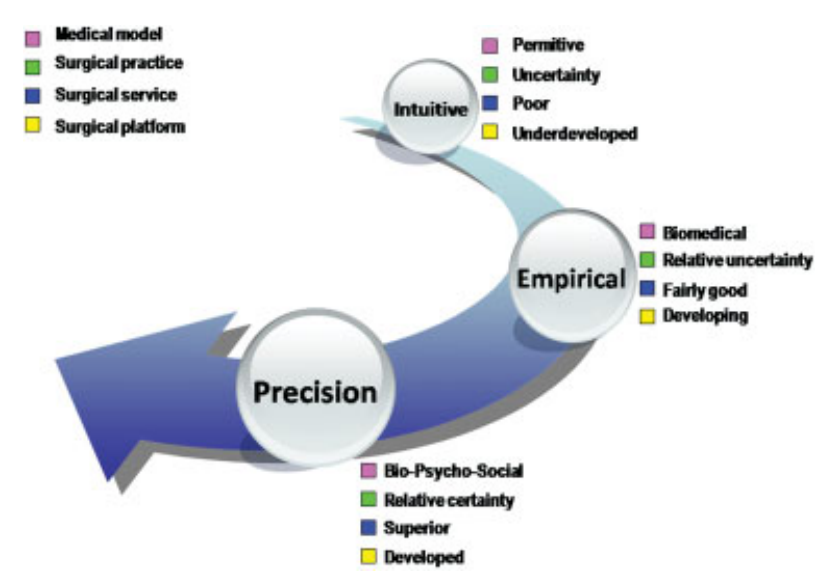

Fig. 1 The schematic evolution of surgery: From intuitive to empirical and moving now toward precision. 


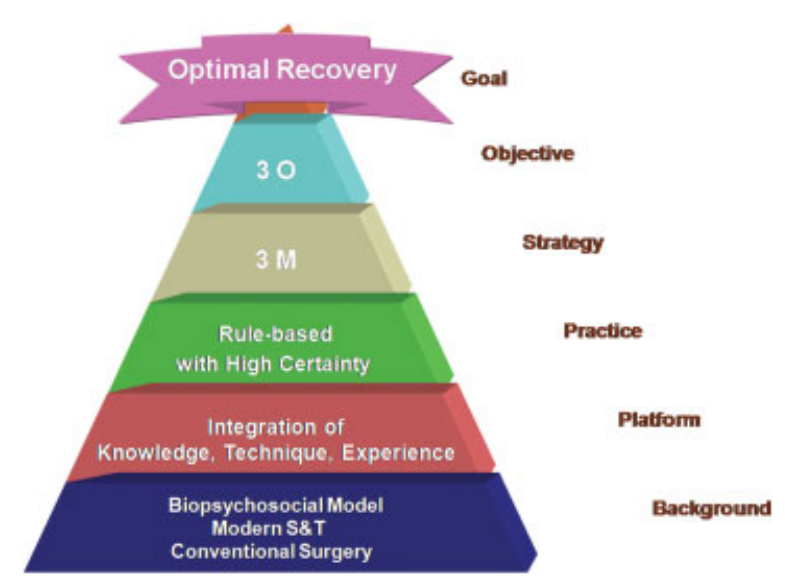

Fig. 2 Pyramidal system of precision surgery. The pyramidal cornerstone of precision surgery consists of state-of-the-art science and technology (S\&T), the bio-psycho-social medical model, and the legacy of conventional surgery. Based on all of these, the platform of precision is established with the integration of available knowledge, techniques, and experience related to surgery. The practice of precision surgery is rule-based with high certainty. The core strategy of precision is to seek and realize a precise balance of " $3 \mathrm{M}$ "-maximal lesion removal, maximal organ sparing, and minimal surgical invasiveness by scientific decision making and accurately controllable surgical intervention. Surgical service as such would realize multiobjective optimization accommodating the " 30 " - therapeutic effectiveness, surgical safety, and minimal invasiveness, and would eventually give rise to the optimal recovery of each patient.

ment will generate an anticipated outcome with total security from error and randomness. The intrinsic uncertainties of medical practice must be appraised, predicted, circumvented, and ultimately attenuated by reasonable decision making. ${ }^{27}$ Distinguished from the former surgical paradigms, precision surgery is able to achieve multiobjective optimization based on its six characteristics: determinacy, predictability, controllability, integration, standardization, and individualization.

\section{Determinacy}

Determinacy of precision surgery based upon scientific determinism represents the ability to generate a desired outcome with a high degree of certainty. This is achieved by identifying the critical sources of uncertainty and controlling their undesired effects in surgical practice. These include multidisciplinary assessment of the patient condition, formulation of treatment strategy, surgical planning, operative manipulation, and perioperative care. When dealing with the remaining yet influential uncertainties, we would attempt to quantify them into probability and risks, and resort to scientific decision making to control the incidence of unfavorable consequences.

In this setting, it is critical for sound decision making and surgical planning to acquire precise information involving the extent of lesion, the safe extent of hepatectomy, etc., through qualitative, quantitative, and real-time evaluation. Advances in hepatic imaging techniques have greatly improved the accuracy in evaluating the extent of the lesion, especially in detecting the minimal foci within liver, hepatic vascular involvement, and extrahepatic micrometastasis. Currently, widely used imaging techniques, such as multidetector computed tomography (CT) and high-field magnetic resonance imaging (MRI) can detect a tumor nodule with a diameter $<10 \mathrm{~mm}^{28}$ Double-enhanced MRI using superparamagnetic iron oxide and gadolinium have increased the diagnostic sensitivity for hepatocellular carcinoma (HCC) of diameter $<1 \mathrm{~cm}$ to $46 \%{ }^{29}$ The current transducer resolution of intraoperative ultrasonography (IOUS) permits the identification of lesions larger than $2 \mathrm{~mm}^{30}$ The benefit of enhanced imaging to the surgeon is obvious and will avoid surgical intervention in the setting of subtle, but disseminated disease, and at the same time, guide more accurate targeting and technique when surgery is indicated.

The critical element in the safety limit of liver resection (SLLR) is the functional capacity of the liver remnant. Conventional methods to evaluate functional liver reserve, such as liver function test and Child-Pugh grading, are mostly qualitative or semiquantitative. The inaccuracy and uncertainty in these tests makes it difficult to determine SLLR. At present, the analysis of multiple parameters, including underlying liver disease, Child-Pugh grading, quantitation of portal hypertension, and the indocyanine green (ICG) retention rate, allow more accurate evaluation of functional liver reserve and determination of the individual SLLR. ${ }^{31-34}$ Combined with imaging-based volumetric measuring, surgeons can accurately assess the functional volume of future liver remnant and determine the resectable extent for safe liver resection. ${ }^{35}$ Recently, we have introduced multimodality imaging technique combining technetium-99m galactosyl serum albumin ( ${ }^{99 m} \mathrm{TC}-\mathrm{GSA}$ ) scintigraphy to quantify the functional capacity of the hepatic segment of interest. For cases with localized variability of parenchymal damage, this method permits demonstration of regional function of the future liver remnant, compared with the conventional methods that only assess total liver functional reserve (data unpublished). This example demonstrates an approach that will increase the level of certainty in the planning of liver resection.

\section{Predictability}

Based on scientific determinism, when causality is precisely evaluated, and attendant processes are accurately controlled, the outcome will be fully predictable. Accuracy in prediction can only be achieved with perfect information and by precise application of the cause-effect rules. Facilitated by advanced science and technology, there is now greater certainty inherent in the acquisition of specific patient information, and in surgical intervention. Compared with the empirical rules of conventional surgery, rules based on current best evidence tend to offer a more accurate interpretation of the causality. If these were integrated rationally, predictability of current surgery could be significantly increased. Precision surgery requires accurate prediction of the consequences of each step in a surgical intervention, the risk of undesirable events, and hence the ultimate treatment outcome.

For example, exploratory laparotomies are frequently performed in conventional liver surgery, whereas accurate preoperative assessment and optimal surgical planning 
should essentially eliminate this event. Computer-assisted surgical planning systems allow comparison and screening of different procedures to select the optimal procedure through "virtual resection." This can be of great help in assessing and predicting the resectability of a liver lesion, especially when lesions involve important anatomic structures and require complex major hepatectomies. ${ }^{4,36}$ We have recently reported that among 65 patients that were initially considered unresectable by experienced surgeons with two-dimensional(2D) imaging, 51 patients were confirmed to be resectable by computer-assisted surgical planning system and finally underwent curative liver resection, with no mortality and major morbidity of only $9.8 \%{ }^{4}$

Based on high-reliability preoperative evaluation, surgeons now can achieve predictability of surgical risk and then formulate the strategy of risk control. For example, according to the relationship between the lesion and the main vessels, we can predict the necessity of vascular resection and reconstruction and the risks of vessel injury and massive bleeding. Aided by individualized assessment of the safety limit for liver resection and accurate analysis of the structural integrity of future liver remnant, posthepatectomy liver failure (PHLF) should be predictable and even avoidable. Currently, in some specialized centers, the incidence of PHLF has declined to $\sim 8 \%{ }^{37,38}$

\section{Controllability}

A high degree of controllability in precision surgery guarantees the anticipated surgical outcome. It relies on both identification of the critical risk factors, and formulation of measures to deal with these factors. In practice, such controllability lies in the high accuracy of operative manipulation, damage control, and risk management.

The liver is one of the largest solid organs, with four sets of entangled structures hidden within parenchyma. In precision surgery, visualization by contemporary advanced techniques has significantly promoted the controllability of surgical intervention and the capacity to circumvent operative risks. As noted above, advanced 2D imaging and digital threedimensional (3D) reconstruction equips the surgeon with real anatomy of the liver including the location of the lesion, the traverse and territory of the vessels, and the spatial relationships between the lesion and vessels. ${ }^{39}$ Intraoperative ultrasonography can help reidentify the location and margin of lesion, position the vessels of interest and the appropriate transection plane, and thereby guide the procedures with controllability. ${ }^{40}$ The segmental boundary can be visualized by portal vein staining technique ( $\mathbf{F}$ Fig. 3) ${ }^{41,42}$ The tumor and its advancing margin can be visualized through in vivo fluorescent imaging. ${ }^{43}$ A real-time navigation system can visualize the lesions and its spatial relationships with important structures throughout the surgical procedure. $^{44}$

Meticulous methods of parenchymal transaction, such as Cavitron ultrasonic surgical aspirator (CUSA), are capable of selectively removing liver parenchyma to expose vessels, enabling surgeons to accurately control the extent of resection, loss of parenchyma, and avoiding vessel injury.
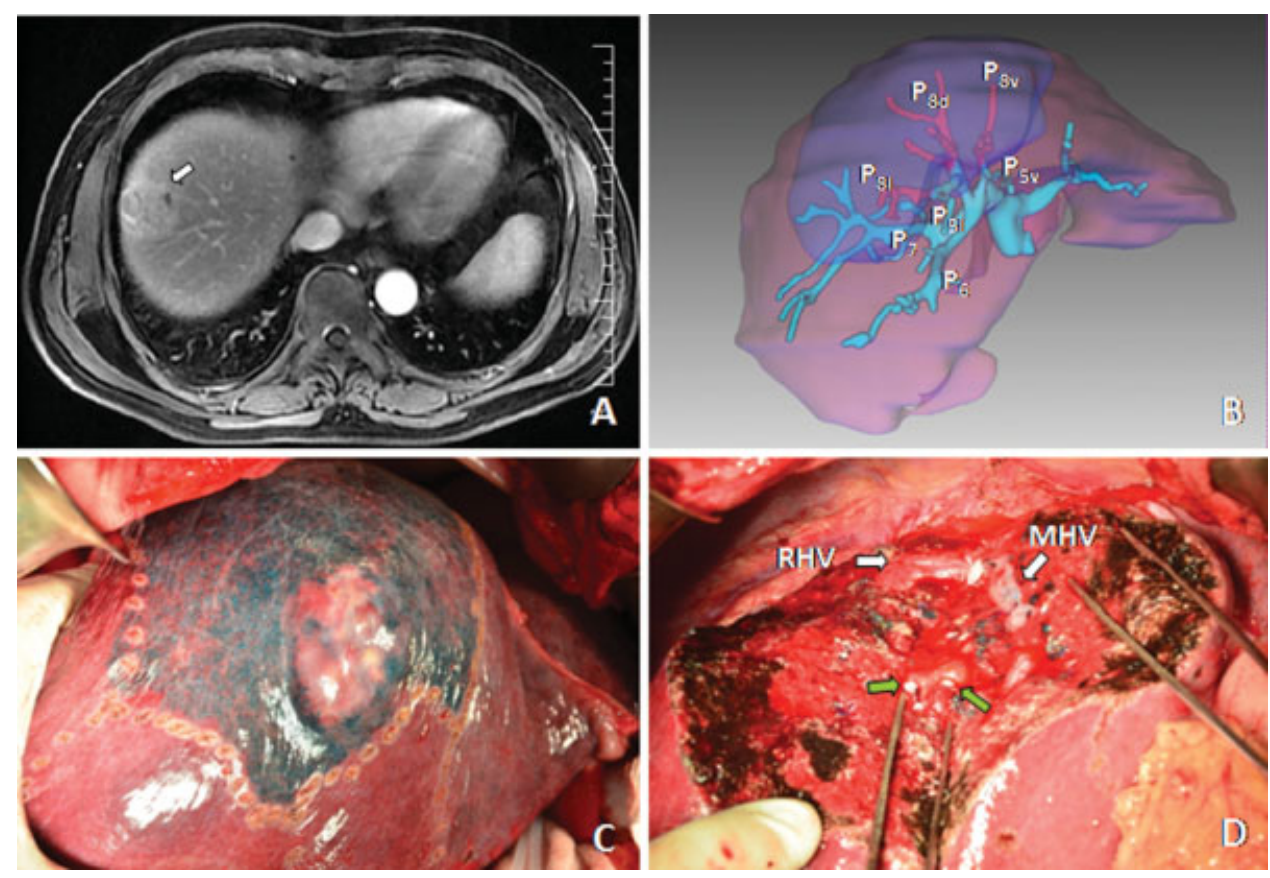

Fig. 3 Regular segmentectomy of $\mathrm{S}_{8}$ guided precisely by methylene blue staining. (A) Computed tomography scan indicated a tumor located within $S_{8}$ (white arrow). (B) Three-dimensional reconstruction shows the intrahepatic distribution of portal branches of $S_{8}$. (C) Intraoperative photograph. Following dissection of the right anterior hepatic pedicle and occlusion of $P_{5 V}, P_{5 L}$, and $P_{6}$, which originate from the right anterior hepatic pedicle, $S_{8}$ was persistently stained by methylene blue injected via the right anterior portal vein, followed by ligation of Glisson pedicle of $\mathrm{S}_{8}$, the territory of $\mathrm{S}_{8}$ was then demonstrated. (D) Intraoperative photograph. $\mathrm{S}_{8}$ was removed, and on the resection plane were the right hepatic vein (RHV; white arrow), middle hepatic vein (MHV; white arrow), and the stumps of Glisson pedicle of $\mathrm{S}_{8}$ (green arrow). 
Precision liver surgery requires control of blood flow through the liver, involving the occlusion of inflow tracts, outflow tracts, and retrohepatic vena cava. Prevention of blood loss is balanced with management of concomitant ischemia-reperfusion injury to the liver remnant. According to a recent systematic review, the mean blood loss in open hepatectomy is below $500 \mathrm{~mL}$, and $320 \mathrm{~mL}$ in laparoscopic hepatectomy, whereas the transfusion rate has been reduced to under $10 \%$ in most specialized centers. ${ }^{45-47}$

\section{Integration}

Integration can increase certainty in surgical practice, and should be patient-centered to optimize the outcome and meet the patients' multidimensional health care needs from a holistic perspective. Precision surgery emphasizes the following: (1) systematic integration of conventional surgical practice with state-of-the-art science and technology to improve the capacity of surgical service; (2) integration of evidence-based rules and experience to optimize surgical decision making; (3) establishing multidisciplinary treatment (MDT) model that complements each specialty's advantages; and (e) rational application of a variety of nonsurgical adjuvant measures to overcome the intrinsic limitations of surgery.

Integration with nonsurgical techniques has brought about an expansion of surgical indications and has served to promote patient safety in liver surgery. Initially, unresectable cases of primary or metastatic neoplasm in the liver may be downstaged via neoadjuvant chemotherapy or interventional therapy to create conditions suitable for radical resection. ${ }^{48,49}$ For cases with unresectable lesion due to a potentially inadequate liver remnant volume, second-stage resection may be feasible if combined with preoperative selective portal vein embolization (PSPVE) to induce hypertrophy of the potential liver remnant. ${ }^{50}$

\section{Standardization}

Quite different from empirical surgery dominated by personal experience, precision surgery relies on rule-based practice to minimize the individual variation among surgeons in intuition, experience, capacity, and cognitive level. The generally applicable rules originated from reliable evidence and/ or specialist consensus can provide surgeons with rational guidance. If these rules evolve into clinical guidelines that apply to the majority of pathologic situations, they will standardize surgical practice in a more powerful manner. These approaches would include standardization of indications using multidisciplinary management, more rigorous adherence to standard surgical procedure, and clinical pathway development to govern the process of care.

Currently, rules of liver surgery have been proposed, including consensus of liver anatomy and terminology of hepatectomies, ${ }^{51,52}$ the staging and classification of various liver diseases, ${ }^{53}$ the principles of liver resection for various benign or malignant liver diseases, decision-making criteria for safe liver resection, ${ }^{31-34}$ and the definition and grading of posthepatectomy complications. ${ }^{54-56}$ Some evidence-based and/or consensus-based clinical guidelines related to surgical management of liver disease have been formulated in the past years. The guidelines for the management of HCC, promulgated by American Association for the Study of Liver Disease (AASLD), European Association for the Study of the Liver (EASL), the Japanese Society of Hepatology (JSH), and Chinese surgical associations, have been used to promote the standardization of the surgical treatment for HCC. ${ }^{57-60}$

We propose three fundamental principles under which precision liver resection should be performed: the anatomical, the physiological, and the pathological. First, the liver remnant must be anatomically integral, with no risk of postoperative ischemia, congestion, or cholestasis; this is the anatomical principle. Second, the liver remnant must be sufficient to guarantee functional compensation-the physiological principle. Third, the procedure of choice must take full account of the biology of the lesion to promote therapeutic effectiveness-the pathological principle. We define anatomical hepatectomy as a procedure dominated by the anatomical principle, which respects the architecture integrity of the liver remnant to avoid isolation of areas of liver from their proper blood vessels and bile ducts, so that complication of such deficiencies could be prevented; with any violation, it should be defined as nonanatomical. A procedure, if evaluated as nonanatomical, should be avoided. On the other hand, a regular hepatectomy aims to precisely remove an integral anatomical unit that bears the lesion, which might be a subsegment, segment, sector, or a full lobe; otherwise, it would be defined as irregular. A regular hepatectomy is preferred in cases with segmental distribution of the lesion, whereas an irregular resection is a more appropriate choice for lesions distributed nonsegmentally, which is a challenge of both pathological and anatomical principles.

\section{Individualization}

Although standardization of the process of care is essential, patients differ markedly in biological and social characteristics mandating surgical care that is precisely tailored to the individual patient. This calls for integration of evidence-based rules, surgeons' experience, as well as patients' individual requirements. ${ }^{61}$ Individualization of diagnostic, prognostic, and treatment strategies is thus accessible in precision surgery. Distinct from the rigidity of rules reflected in standardization, precision surgery also highlights individualization and flexibility in the application of rules.

In liver surgery, the adaptation is demonstrated in the choice of the procedure of hepatectomy, surgical access, method of liver blood control, technique for parenchyma transaction, etc., for the specific patient. Regular hepatectomy is preferred in cases with a sufficient functional liver remnant, segmental distribution of the lesion, and cases calling for obligatory removal of the involved proper pedicle. On the other hand, irregular resection is a more appropriate choice for tumor with limited or no infiltration, peripheral lesions that do not involve main vessels, or in patients with marginal functional liver reserve. For example, regular hepatectomy has been recommended for cystic biliary dilatations involving segmental duct of liver (third-order hepatic duct). But the 
extent of cyst-involved segments vary greatly in each patient, and the surgical procedure may be individualized. ${ }^{62}$

\section{Precision in Liver Surgery}

Advances in liver surgery have reduced blood loss, and a decline in morbidity and mortality ${ }^{63-66}$ However, the mortality rate in extended liver resection, the rate of curative resection for liver malignancies, and postoperative long-term survival are still far from satisfactory. ${ }^{67}$ Therefore, we hope the concept of precision will help propel liver surgery into a brand new era.

\section{The Strategy for Precision Liver Surgery}

In liver surgery, the effectiveness lies in the eradication of target lesion, safety in sufficient compensation of liver remnant, and minimal invasiveness in attenuating surgical trauma without compromising effectiveness and safety. The strategy of precision liver surgery is to seek the precise balance among 3M: maximizing the removal of the target lesion, maximizing the functional liver remnant, and minimizing surgical invasiveness.

Strategy for Maximizing the Removal of Target Lesion Maximizing the removal of the target lesion is the premise of surgical therapeutic effectiveness. Target lesion refers to the partial or entire pathology of the intra- and extrahepatic region, the entire removal of which can achieve the goal of locoregional control, elimination of symptoms, and even cure of the disease. The extent of the target lesion varies in light of the underlying disease (-Fig. 4).

\section{Precise Evaluation of the Target Lesion}

Maximizing the removal of target lesion begins with an accurate evaluation for the extent of the target lesion, including the detected lesion itself, along with the potential involvement beyond detection yet within deduction. Precision liver surgery integrates high-resolution imaging, such as multidetector CT, high-field MRI, contrast-enhanced ultrasound, and IOUS to detect the minimal foci and to accurately assess the tumor stage. ${ }^{28-30}$ Morphology is modulated by an understanding of the expected biological behaviors of the tumor to surmise its pathologic boundary. Although in current practice, a preoperative examination should accurately predict resectability, laparoscopy may be used for exploration and staging to exclude intraperitoneal metastasis or regional advanced malignancies. ${ }^{68,69}$

\section{Downstaging of Unresectable Tumors}

For some unresectable hepatobiliary malignancies, downstaging treatment has been applied to create conditions for radical resection. In recent years, several studies suggest that
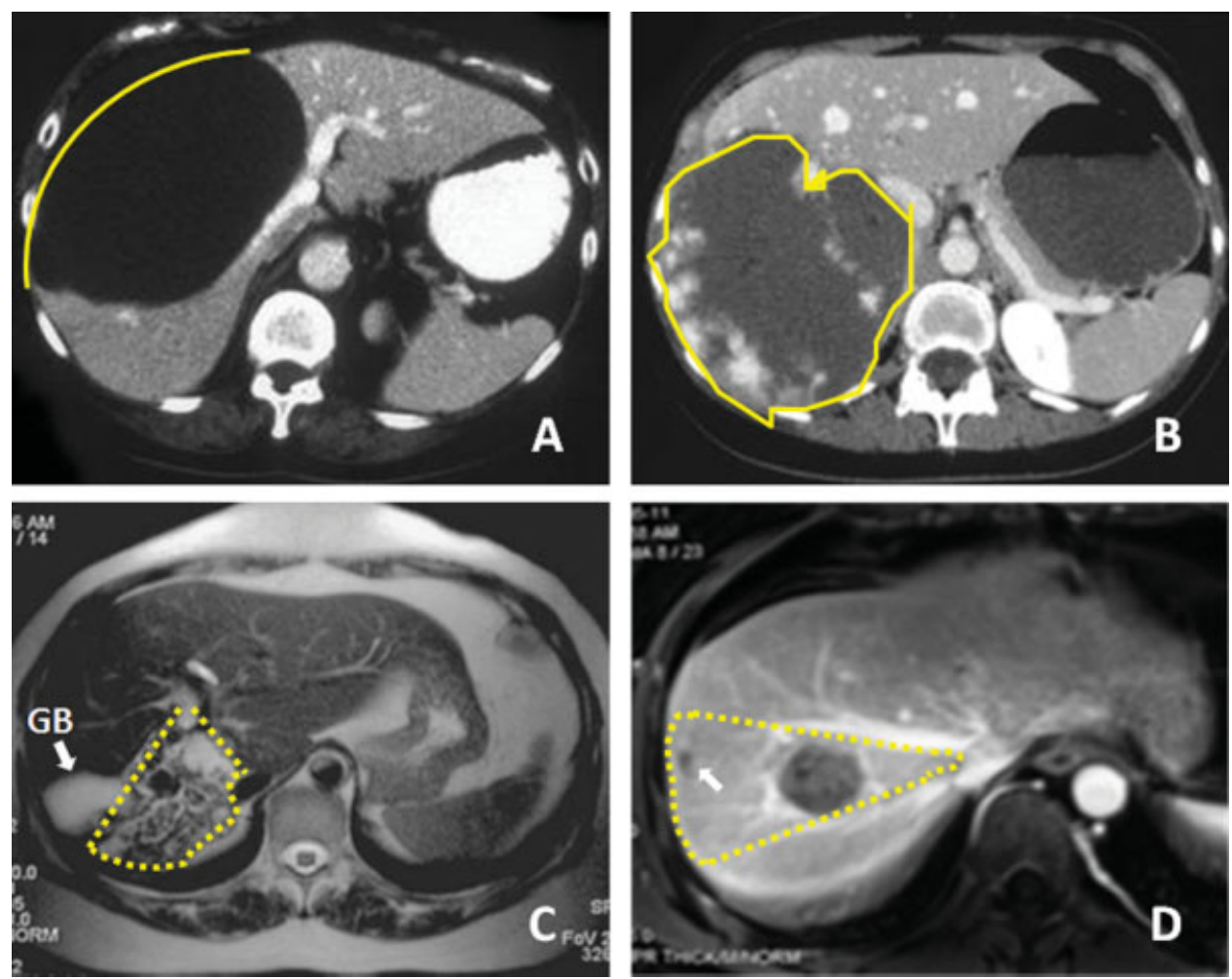

Fig. 4 Determination of the extent of the target lesion (outlined by the yellow line). In cases with simple giant hepatic cysts, it is only necessary to remove the outwardly protruding wall of the cysts $(A)$. In cases with hepatic benign tumor, the target lesion is defined as the tumor itself. Tumorectomy along its boundary is preferred for hemangioma (B). For some benign cholangiopathy that involves the segmental hepatic ducts or more proximal branches, such as hepatolithothiasis or the cystic dilation of hepatic ducts, the target lesion includes the involved hepatic ducts and their draining segments. Target lesion of a localized-type hepatolithothiasis is shown in (C). GB, gallbladder. For malignant hepatic tumors with a propensity for portal invasion and metastasis, the target lesion would include the tumor itself and the potentially invaded peritumoral tissue. Resection of the tumor-bearing segment with minimal metastases (white arrow) is preferred in this advanced hepatocellular carcinoma (D). 
for previously unresectable colorectal liver metastases, $\sim 22.5 \%$ of the cases can eventually undergo radical resection after neoadjuvant chemotherapy. ${ }^{49}$ For those HCC cases that cannot tolerate primary radical resection, transcatheter arterial chemoembolization (TACE) and internal radiation therapy can reduce the size and number of tumors to facilitate secondary radical resection. Some studies indicate that $\sim 8$ to $18 \%$ of the previously unresectable HCC cases receive successful secondary resection following downstaging treatment, with a 5-year survival ranging from 24.9 to $57 \%$. ${ }^{48}$

\section{Oncologic Principles during Surgery}

The major principle of oncologic resection is to eliminate the target lesion en bloc with an adequate tumor-negative margin using tumor-free approaches. ${ }^{70}$

For advanced HCC, ideally, the closest margin from the tumor edge should be at least $1 \mathrm{~cm}$ due to its propensity of local infiltration. Because portal dissemination is frequently observed, the preferred procedure should be regular resection of the tumor-bearing segment. We advocate that the optimal resection margin should include the boundary of the tumor-bearing segment with at least $1-\mathrm{cm}$ margin distance from the tumor's edge in a regular hepatectomy. There is strong evidence in favor of anatomical resection for HCC with a diameter of 2 to $5 \mathrm{~cm}$ although the survival benefit for HCC greater than $5 \mathrm{~cm}$ has been questioned. ${ }^{71,72}$ For colorectal liver metastases, the current evidence does not favor anatomical hepatectomy over nonanatomical hepatectomy, with a preferred resection margin exceeding $1 \mathrm{~cm} .{ }^{73-75}$ However, these issues remain controversial.

For both primary and metastatic liver tumor, the surgical outcome is closely related to its regional lymph node metastasis, but the necessity of lymphadenectomy and its extent vary in different malignancies. ${ }^{76-78}$ The cholangiocyte-originated malignancies, including hilar cholangiocarcinoma, intrahepatic cholangiocellular carcinoma, and advanced gallbladder carcinoma, are characterized by a propensity to regional lymph metastasis, thus locoregional lymphadenectomy should be an integral component of the resection.

For hepatobiliary malignancies involving major hepatic vasculature, hepatectomy combined with vascular resection and reconstruction can substantially increase the rate of curative resection and the overall survival. ${ }^{79-81}$ For advanced hilar cholangiocarcinoma, hepatectomy combined with simultaneous resection and reconstruction of portal vein and hepatic artery has promoted the $\mathrm{R}_{0}$ resection rate to $66 \%$, and the 5-year survival rate to $30.3 \%{ }^{79}$

To avoid iatrogenic spread of tumor, en bloc resection technique and the no-touch principle should be followed. The orthotopic liver resection, also known as anterior approach hepatectomy, is recommended for the resection of huge liver tumors. $^{82}$

\section{Strategy for Maximizing the Functional Liver Remnant}

The functional volume of the liver remnant and its vascular integrity are crucial to postoperative functional compensation and surgical safety.

\section{Individualized Assessment of the Safety Limit for Liver Resection}

The resectable volume is limited by the extremity of the liver functional compensation. The safety limit for liver resection depends on the minimal functional liver volume required for body needs, ${ }^{83}$ which we named the essential functional liver volume (EFLV, $V_{E}$ in formulas). The EFLV is based mainly on the standard liver volume ( $\mathrm{SLV}, \mathrm{V}_{\mathrm{S}}$ in formulas) and the status of the functional liver reserve.

$$
\begin{aligned}
& V_{\mathrm{E}}=\beta+\mathrm{V}_{\mathrm{S}} \\
& \beta=\mathrm{V}_{\mathrm{E}} / \mathrm{V}_{\mathrm{s}}
\end{aligned}
$$

The SLV of each patient is relatively constant, and it is estimated from the human body surface area or body weight. ${ }^{84} \beta$ here is a patient-specific coefficient that is a function negatively related to the functional liver reserve.

Because the EFLV cannot be determined in an absolutely precise way for a given individual, the true value of $\beta$ cannot be accessed. In practice, with the inferred EFLV and estimated SLV, we can use the ratio of EFLV to SLV (standardized ratio of EFLV, $R_{S E}$ in formulas) as the estimated value of $\beta$.

$$
\begin{aligned}
& R_{S B}=V_{E} / V_{S} \\
& V_{E}=R_{S E} \cdot V_{S}
\end{aligned}
$$

The SLLR ( $V_{S R}$ in formulas) refers to the maximal resectable liver volume with only the EFLV preserved, which equals to the total liver volume (TLV, $\mathrm{V}_{\mathrm{T}}$ in formulas) subtracted the EFLV.

$$
\mathrm{V}_{\mathrm{SR}}=\mathrm{V}_{\mathrm{T}}-\mathrm{V}_{\mathrm{F}}=\mathrm{V}_{\mathrm{T}}-\mathrm{R}_{\mathrm{SF}} \cdot \mathrm{V}_{\mathrm{S}}
$$

And the prerequisite for a safe liver resection is that the functional volume of liver remnant (RFLV; $V_{R}$ in formulas) is higher than EFLV, which means that the ratio of RFLV to SLV (standard ratio of RFLV, $R_{S R}$ in formulas) is higher than $R_{S E}$.

$$
\begin{gathered}
V_{\mathrm{K}} \geq \mathrm{V}_{\mathrm{E}}=\mathrm{R}_{\mathrm{SE}} \cdot \mathrm{V}_{\mathrm{S}} \\
\mathrm{V}_{\mathrm{K}} / \mathrm{V}_{\mathrm{S}}=\mathrm{R}_{\mathrm{Sk}} \geq \mathrm{R}_{\mathrm{SH}}
\end{gathered}
$$

Currently, there are some decision-making criteria for safe liver resection, as proposed by Makuuchi ${ }^{31}$ and Clavien, ${ }^{33}$ which are useful in clinical practice. In our center, we consider the presence of cirrhosis, Child-Pugh functional classification, and ICG $\mathrm{R}_{15}$ to assess the functional liver reserve and quantify the safety limit of liver resection with $\mathrm{R}_{\mathrm{SE}}$ and establish a decision tree for liver resection (-Fig. 5). ${ }^{34}$ Retrospective studies show that, compared with the two criteria mentioned above, our decision tree expands the indications for liver resection, with no increase in posthepatectomy liver failure. ${ }^{85}$ This algorithm has become the Chinese consensus.

Measures to Increase the Functional Volume of Liver Remnant If the predicted RFLV is less than the EFLV, or $R_{S R}<R_{S E}$, methods must be applied to enlarge the functional liver remnant. It is therefore important to eliminate any reversible factors of liver injury such as obstructive jaundice, fatty change, and chemotherapy-induced liver injury. ${ }^{86,87}$ For 


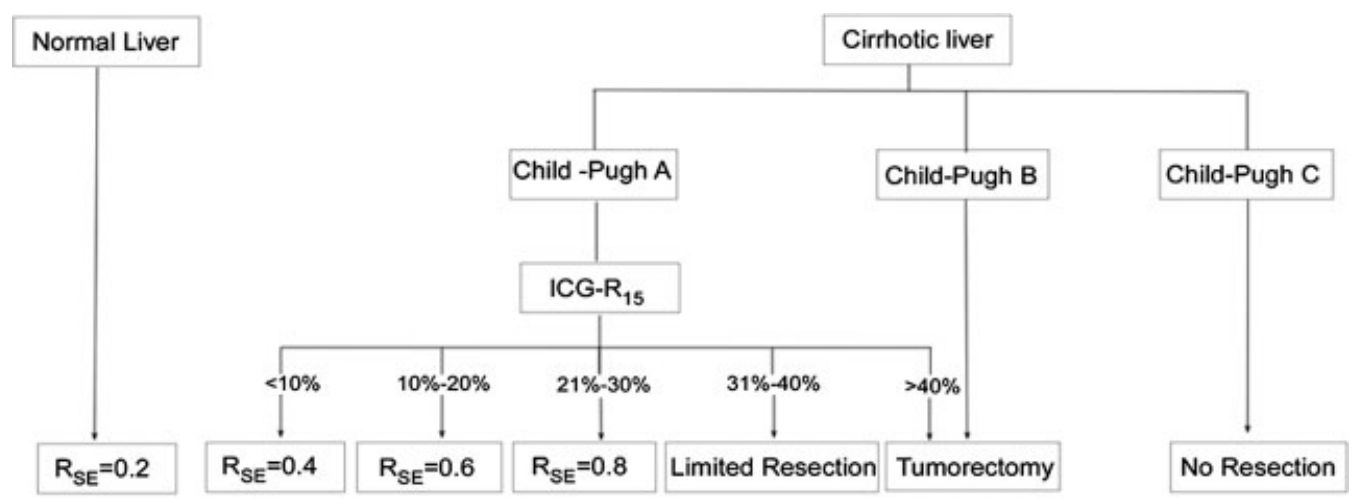

Fig. 5 Chinese consensus for the assessment of safe limits for liver resection. The general consensus is that $R_{S E}$ should be 0.20 for normal liver. For the cirrhotic cases with liver function in Child A class, if indocyanine green (ICG) $R_{15}$ is less than $10 \%$, $R_{S E}$ should be 0.40 . If ICG $R_{15}$ ranges from 10 to $20 \%, R_{S E}$ should be 0.60 , and 0.80 for cases with ICG $R_{15}$ between $21 \%$ and $30 \%$, respectively. Patients can only undergo limited liver resection with ICG $R_{15}$ ranging from 31 to $40 \%$. When ICG $R_{15}$ is over $40 \%$ or liver function is in Child B class, the tumorectomy becomes the only suitable procedure. Child C class is a contraindication for surgery.

cases with chemotherapy-induced liver injury, liver resection is delayed at least 6 to 8 weeks after chemotherapy to allow the recovery of liver function. ${ }^{88}$

Preoperative selective portal vein embolization is one of the effective approaches to induce hypertrophy of the potential liver remnant. Published work indicates that the mean hypertrophy rate of the future liver remnant was $37.9 \%$, 4 weeks following PSPVE, and $80 \%$ of patients were able to withstand planned hepatectomy. ${ }^{50}$ In cases with insufficient hypertrophy following PSPVE, in situ liver split as proposed by the Berlin team may permit a marked and rapid hypertrophy of functional liver tissue. ${ }^{89,90}$

Parenchymal sparing surgery will enhance the safety of resection, particular in the surgery of metastases in which repeat resection may be required. Radiofrequency ablation is complementary following major resection to eliminate residual foci within liver remnant, which can save more liver parenchyma. ${ }^{91}$

\section{Preservation for the Structural and Functional Integrity of Liver Remnant}

Vascular and biliary integrity are prerequisites for function of the liver remnant. Although daunting and technically demanding, optimal preoperative evaluation, surgical planning, and operative manipulation permits excision and reconstruction of involved vessels without compromising oncologic principles. Vascular reconstruction is indicated when the functional volume of liver remnant is insufficient or $\mathrm{R}_{\mathrm{SR}}<\mathrm{R}_{\mathrm{SE}}$, or the probability of ischemic necrosis within liver remnant is substantial. In complex liver resection, a computer-assisted surgical planning system can help identify vascular variations and the hepatic territory at risk for ischemia, thereby refining the indications for vascular reconstruction.

In addition to the arteries, portal veins and biliary pedicles, the outflow of the liver may require reconstruction particularly if remnant volume is marginal. This may include the reconstruction of the vena cava if resection of the retrohepatic inferior vena cava (IVC) impeded the outflow of the liver and/or kidney. ${ }^{92,93}$ However, in cases of complete IVC obstruction below the hepatic venous convergence with full collateral compensation, IVC reconstruction may be avoided (-Fig. 6).

The control of ischemia-reperfusion (I/R) injury is paramount and inflow occlusion during resection must be managed to attenuate this phenomenon. Current investigations have established that controlled low-inflow state with isolated occlusion of the portal vein can significantly reduce $\mathrm{I} / \mathrm{R}$ injury to liver remnant with no increase in blood loss, compared with the Pringle maneuver. ${ }^{94-97}$ In our center, this method is preferred and we apply isolated occlusion of portal vein or no flow occlusion in cases with severe parenchymal damage and critical liver remnant as well as graft harvesting for live-donor-liver transplantation.

\section{Strategy for Minimizing Surgical Invasiveness}

Through a series of measures over the course of the entire perioperative period, we can reduce the collective effects of local, systemic, and psychological trauma induced by liver resection.

\section{Control of the Intraoperative Blood Loss}

Blood loss is an independent risk factor closely related to the early and long-term prognosis for hepatectomy. ${ }^{98,99}$ Anesthetic strategies such as controlling central venous pressure (CVP) $\left(<5 \mathrm{~cm} \mathrm{H}_{2} \mathrm{O}\right)$, reduces venous blood loss during parenchymal transection. ${ }^{100}$ A variety of techniques of inflow control, such as the Pringle maneuver, dissection and occlusion of hepatic artery and portal vein, or selective semihepatic vascular occlusion, can effectively control bleeding during liver resection. For some huge central tumors involving the convergence of hepatic veins or IVC, total vascular exclusion (TVE) with or without extracorporeal venous bypass can be an option. ${ }^{101,102}$ If complex vascular resection and reconstruction is unable to be completed in situ with controllable bleeding, the liver surgery can be performed ex vivo with excision and reimplantation of the liver after removal of the tumor. ${ }^{103,104}$ 

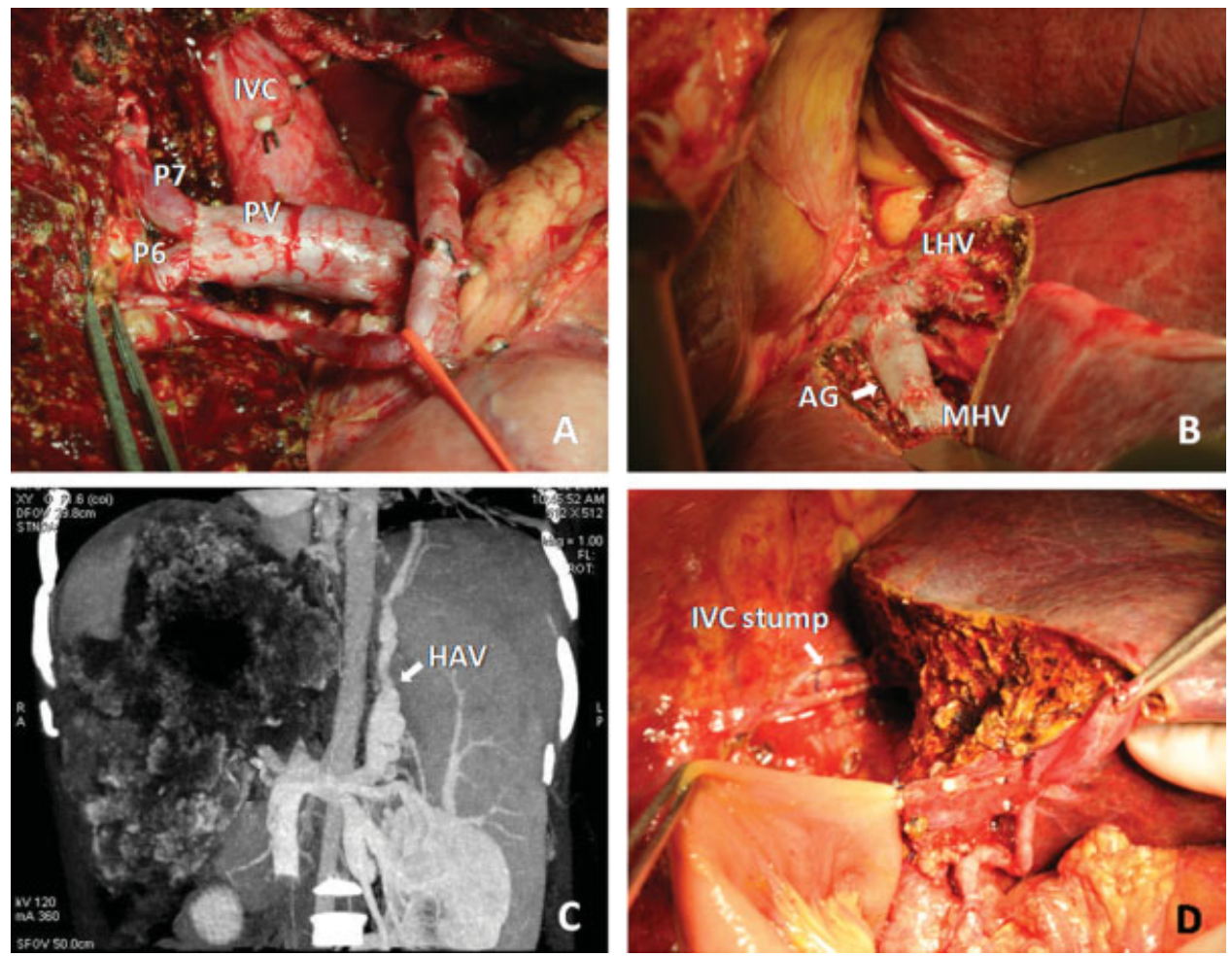

Fig. 6 Decision for vascular reconstruction in liver resection. (A) Left trisectionectomy combined with resection of the right branch and confluence of the portal vein was performed for a case of hilar cholangiocarcinoma, followed by reconstruction of the main trunk of the portal vein with the stumps of $\mathrm{P}_{7}$ and $P_{6}$. (B) Subsegmentectomy for $S_{4 a}$ with resection of the involved middle hepatic vein was performed in a case with hepatocellular carcinoma, followed by reconstruction of the middle hepatic vein with an autologous graft (AG) from the internal jugular vein. LHV, left hepatic vein, MHV, middle hepatic vein. (C,D) This is a case of hepatic alveolar echinococcosis. Computed tomography scan shows the retrohepatic inferior vena cava (IVC) was completely invaded with the hemiazygos vein open (HAV; white arrow). An extracorporeal right trisectionectomy with resection of the retrohepatic IVC (white arrow) was performed, followed by autologous liver transplantation with the left lateral lobe. The left hepatic vein was reconstructed with the right portal vein, while the IVC was not reconstructed due to collateral compensation from HAV.

\section{Attenuation of Tissue Damage}

Surgeons need to operate under the general principles of gentle manipulation and delicate dissection to minimize surgical invasiveness. The minimally invasive techniques of liver parenchymal transection, such as the fine clamp-crushing technique, CUSA, and harmonic scalpel should be rationally utilized to reduce damage to the liver parenchyma and vascular within the liver remnant.

\section{Minimal Access Surgery}

Laparoscopic hepatectomy is a minimally invasive approach, but with limited control of the accuracy of the manipulation limiting its application. Established indications include single $\mathrm{D} \leq 5-\mathrm{cm}$ tumor located in segments $2,3,4,5$, and $6 .{ }^{105}$ Robotic surgical systems have enabled the surgeons to perform controlled hepatectomy with high accuracy in dissection of the hilum, hepatocaval dissection, endoscopic suturing, and microanastomosis. ${ }^{106}$ Our preliminary experience shows that robotic-assisted laparoscopic anatomical hepatectomy can be performed precisely with a much lower complication and conversion rate than conventional laparoscopic hepatectomy. ${ }^{20}$

\section{Accelerate the Recovery}

A group of interventions grouped under the concept of "fast track" surgical management may be applied to liver surgery including optimal analgesic strategies, appropriate intravenous infusion, early-stage enteral nutrition, and aggressive early mobilization. ${ }^{107,108}$ These considerations are of increased importance in the subset of patients at high risk for PHLF, including those with small-sized liver remnant, prolonged vascular occlusion, or massive intraoperative bleeding.

\section{Decision Making in Precision Liver Surgery}

Conceptually, if each of the $3 \mathrm{M}$ of surgery approaches the level of certainty, each of the 30 of surgical service would be optimized. To incorporate determinacy in practice, surgical decision making on a single dimension of the $3 \mathrm{M}$ is grounded on acquisition of sufficient certainty, and management of the remnant yet influential uncertainties. We emphasize that, in the face of imperfect information, the certainty needed for a sound decision, while diminished, is adequate for safe and effective practice. For example, for a relatively simple case such as a benign neoplasm with limited involvement, imaging with high resolution may not be necessary for decision making. On the other hand, high-quality imaging might be essential to support a decision to observe a lesion on the supposition that it is malignant. Precision in surgery may be enhanced through the use of decision analysis, in which nodes of uncertainty are populated with estimated probabilities based on real data. The identification and management 
of critical uncertainties involved in a defined objective of 30 may be quantitatively arranged into the relative probabilities of a set of possible states, each exerting different, but definite effect on the outcome of surgical intervention. A series of feasible surgical options may then be generated in accordance with the quantified uncertainties, each with a predictable prognosis. Determined by utility analysis, the alternative fulfilling the anticipated outcome would be preferred. Thus, uncertainty and its potential undesired consequences are mitigated. As an example, the uncertainty of the extent of invasion of a liver malignancy may be arranged as the aggregate probabilities of a set of possible pathologic states based on the tumor factors. This measured uncertainty would generate a series of feasible procedures with various extents of resection yielding different yet definite prognosis. In the absence of a contraindication to a more radical resection, the alternative that removes the target lesion with minimal risk of recurrence is preferred.

However, in the practice of liver surgery, the overall outcome is not the simple sum of effects on each dimension due to their interaction of other factors. In fact, a single solution that optimizes all of the 30 is uncommon because this problem involves multiobjectives along with constraints on what combinations of those 30 are attainable, especially with the presence of uncertainties. We have to solve this multiobjective optimization problem with strategies to seek a precise balance of $3 \mathrm{M}$, and ultimately to achieve the unity of 30. The most commonly applied method is to convert the original problem with three objectives into a single-objective optimization problem. This is called a scalarized problem. For a specific patient, if it is obvious that one $M$ were least important or can be evaluated with highest degree of certainty, it can be predefined or transformed into a constraint. Then with a designated second $\mathrm{M}$, we evaluate each alternative decision among the varying third $\mathrm{M}$ via quantifying and predicting the definite probability of occurrence of the undesired event and its possible consequences. Thus, a singleobjective optimization problem is formulated, whose optimal solution becomes the solution to the original three-objective optimization problem. With different parameters for the scalarization, different alternative solutions are produced. The one that could achieve the anticipated outcome with the most controllable risk would be taken as the treatment of choice.

Although currently in practice, our process for ranking the alternatives is judgment-based, and sometimes nonquantitative; in principal, it can use an aggregate objective function to rate the alternative set of predicted outcomes. We believe that in the future, digital assistance with an established mathematical model might be developed to carry out the task of multiobjective optimization.

\section{Case Demonstration for Decision Making and Surgical Planning in Precision Liver Surgery}

\section{Decision Making}

Here we will use a case of Caroli disease to illustrate our clinical decision-making process and surgical-planning pro- gram under the theory and methodology of precision (-Fig. 7). The patient was a 26-year-old woman, and suffered from recurrent epigastric pain and fever for 5 years. The diagnosis was established by clinical symptoms and imaging, admitted to Chinese PLA General Hospital on May 20, 2008. For further evaluation, CT and magnetic resonance cholangiopancreatography and $3 \mathrm{D}$ reconstruction showed that most of the liver was involved by cystic dilation of peripheral bile ducts, except the common trunk of the biliary tree, $S_{2}, S_{3}$, and part of $S_{5}, S_{6}$, the Spiegel lobe, and caudate process of $S_{1}$. There was no evidence of mesenchymal fibrosis or portal hypertension, and the liver functional tests were normal. Indocyanine green clearance at 15 minutes was $10 \%$. The patient's physiological status was class I according to her American Society of Anesthesiologists (ASA) score.

The treatment options included liver transplantation, aggressive hepatectomy with/without PSPVE or combined with auxiliary transplantation, and conservative treatment. In decision making for this case, we applied the method of scalarization.

\section{Maximize the Removal of the Target Lesion}

Caroli disease is a benign disease, but has propensity for malignant transformation. ${ }^{109}$ Aside from this disease, the patient was thought to have a long life expectancy. Considering her quality of life, disease-free survival was deemed to be the priority. Based on current best evidence, elimination of the target lesion in Caroli disease would provide a favorable long-term outcome. The necessity of lesion removal could then be defined with certainty. The surgical options that would not achieve elimination were excluded.

\section{Minimize the Surgical Invasiveness}

The patient was young and robust with a relatively high tolerance of surgical trauma. This dimension would then be scalarized into a constraining condition due to its least importance. If performed with refined bleeding and trauma control, major intervention would be tolerated; a less invasive approach, laparoscopic hepatectomy, was excluded due to its relatively poor accuracy and controllability.

\section{Maximize the Functional Liver Remnant}

After scalarization, the three-objective optimization problem is reduced to this one. Although the initial evaluation indicated bilobar involvement posing the consideration that the only long-term curative procedure would be liver transplantation. ${ }^{110}$ However, due to the lifelong consequences of immunosuppression as well as the problems associated with access to transplantation, it was determined that liver transplantation, total or auxiliary, should be avoided if possible. Though the process is bilobar, advanced imaging identified a territory of the liver that was uninvolved that would be the destined surgical remnant. The theory and techniques of precision enabled us to identify the aggressive hepatectomy as the optimal surgical solution that would balance surgical risk with the probability of long-term benefit. 


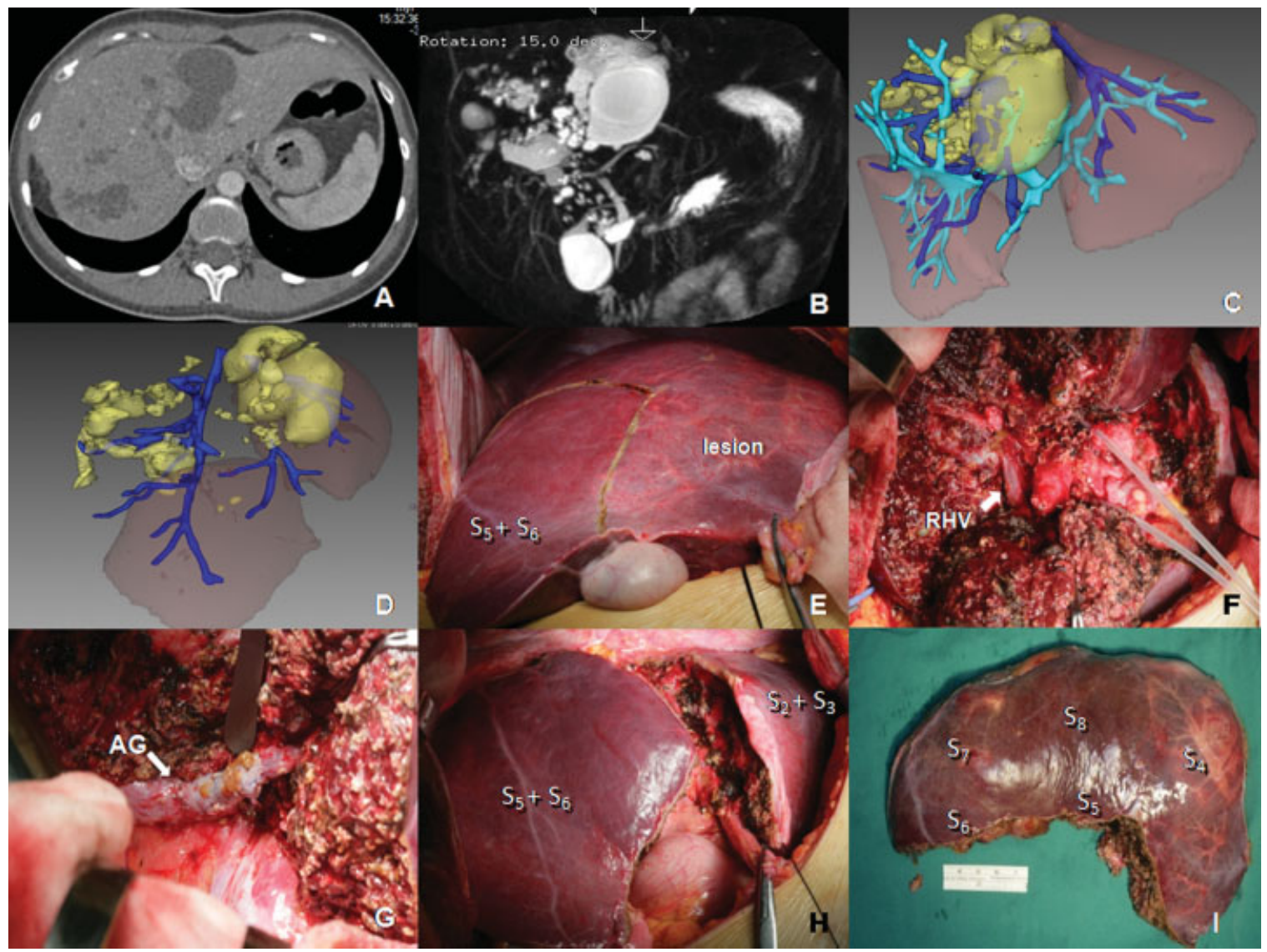

Fig. 7 Illustration of preoperative evaluation and surgical procedure in a patient with Caroli disease. (A) Preoperative computed tomography imaging. (B) Preoperative magnetic resonance cholangiopancreatography. (C) Three-dimensional (3D) reconstruction indicated that most of the liver is involved by cystic dilation of peripheral bile ducts, except the trunk of the biliary tree, $S_{2}, S_{3}$, part of $S_{5}, S_{6}$, the Spiegel lobe, and caudate process of $S_{1}$. (D) 3D reconstruction analysis revealed that drainage of $S_{5}, S_{6}$ was dominated by the right hepatic vein (RHV). (E) Intraoperative view of the extent of the lesion and the uninvolved portion of $S_{5}, S_{6}$. $(F)$ RHV was found after transection on the irregular plane within $S_{5}$, $S_{6}$ between the involved portion and normal parenchyma. (G) Outflow of $S_{5}, S_{6}$ was reconstructed with autologous graft (AG) of resected RHV. (H) There was no sign of congestion or ischemia in the liver remnant. (I) The resected specimen.

For this patient, the SLV was calculated as $1292 \mathrm{~mL}$. With no underlying disease, preserving $20 \%$ of the SLV was required, which was an EFLV of $258 \mathrm{~mL}$. However, the total volume of uninvolved segments $\left(\mathrm{S}_{2}+\mathrm{S}_{3}\right)$ was $208 \mathrm{~mL}$ (16.1\%), which was less than the EFLV. A relatively simple resection option, right trisectorectomy was too risky because of the small remnant. Preoperative selective portal vein embolization to enlarge the remnant was an option, but was not technically possible because the right portal vein was too thin. Thus, it was necessary to design a more complex procedure that would preserve the uninvolved portions of $S_{1}$, $S_{5}$, and $S_{6}$, increasing the estimated functional liver remnant volume (FLRV) to $31.5 \%$ of SLV. The high complexity of this surgical procedure entails a risk of massive bleeding and postoperative liver failure. The elements favoring this approach include surgical tolerance of the patient, and that availability of advanced surgical and anesthetic capability for a complex resection. The surgical procedure chosen was therefore a regular hepatectomy of $\mathrm{S}_{4}, \mathrm{~S}_{7}$, and $\mathrm{S}_{8}$, and irregular hepatectomy of $S_{1}, S_{5}$, and $S_{6}$.

\section{Surgical Planning}

Determine the Region of Obligatory Liver Resection

Diffuse involvement of $S_{4}, S_{7}$, and $S_{8}$ demonstrated the necessary for total removal, and partial involvement of $S_{1}$, $\mathrm{S}_{5}$, and $\mathrm{S}_{6}$ suggested irregular resection should be performed.

\section{Determine the Obligatory Extent of Liver Preservation}

In this case, it included the uninvolved segments $\left(\mathrm{S}_{2}, \mathrm{~S}_{3}\right)$ and the uninvolved portion of $S_{1}, S_{5}$, and $S_{6}$.

Determine the Volume, Structure, and Function of the Potential Liver Remnant

The potential liver remnant included $\mathrm{S}_{2}, \mathrm{~S}_{3}$, and the preservable portion of $S_{1}, S_{5}$, and $S_{6}$. Its volume was assessed as $407 \mathrm{~mL}$ without underlying disease, which was sufficient for compensation. The planned resection would not affect the inflow and outflow of $S_{1}, S_{2}$, and $S_{3}$. But drainage of $S_{5}$ and $S_{6}$ is dominated by the right hepatic vein (RHV), which had to be removed along with the lesion. 
Determine the Optionally Resectable Extent and the Rational Resection Extent

In this case, to maximize preservation of normal liver parenchyma, the region of obligatory resection was determined as the rational resection extent.

\section{Determine the Optimal Procedure for Hepatectomy and the Parenchyma Transection Plane}

The transaction plane needed to be optimized via virtual hepatectomy with the computer-assisted surgical-planning system. On the left, a regular transection plane between $S_{4}$ and $S_{2}, S_{3}$ was designed; on the right inferior, an irregular plane was designed within $S_{5}$, and $S_{6}$ between the involved portion and normal parenchyma.

\section{Determine the Vessels to Be Resected and Reconstructed}

The Glisson pedicles of $\mathrm{S}_{4}, \mathrm{~S}_{7}$, and $\mathrm{S}_{8}$ would be transected, as would the short hepatic vein, RHV, and middle hepatic vein (MHV). Absence of the inferior right hepatic vein and planned resection of the RHV would render RHV reconstruction obligatory to give $S_{5}$ and $S_{6}$ an outflow tract

\section{Determine the Surgical Risk and Its Management}

This was an aggressive hepatectomy, with a marginal liver remnant. The estimated $\mathrm{R}_{\mathrm{SR}}$ was just slightly higher than the $\mathrm{R}_{\mathrm{SE}}$. The transection plane would involve many major vessels and would leave a huge raw surface; there was high risk of massive bleeding and insurmountable surgical stress, which might lead to PHLF. The procedure must be carefully designed to minimize $I / R$ injury to the intended liver remnant and limiting blood loss. In this case, $S_{2}$ and $S_{3}$, which were of paramount importance to recovery, were designated not to undergo ischemia. Reconstruction of the RHV made occlusion of $S_{5}$ and $S_{6}$ obligatory, yet the duration needed to be minimized. Intraoperatively, we planned to apply IOUS as guidance to ensure controllability and manipulative accuracy and prevent vascular injury.

\section{Determine the Surgical Process, Operative Access, and Critical Techniques}

In this case, we elected to emphasize certain key points: (1) sparing the normal liver parenchyma; (2) reconstruction of vascular integrity of $S_{5}$ and $S_{6}$; (3) control of blood loss. Cavitron ultrasonic surgical aspirator, as a preferred fine transection technique, would minimize the loss of normal parenchyma. Parenchyma was transected without portal occlusion to reduce I/R injury. Continuous low CVP $(<5 \mathrm{~cm}$ $\mathrm{H}_{2} \mathrm{O}$ ) was implemented to control blood loss. During RHV reconstruction, inflow occlusion of $S_{5}$ and $S_{6}$ would be necessarily applied, and the resected RHV was used for autologous reconstruction.

Determine the Need for Adjuvant Therapy and Perioperative Management

After aggressive hepatectomy, hypervolemia would cause congestion in the liver remnant. Control of circulation volume would be crucial to prevent small-for-size syndrome. Homoiostasis must be maintained to ensure liver regeneration.
Extra attention must also be paid to prevention and management of complications.

The procedure was performed strictly according to the preoperative protocol on May 26, 2008, the total blood loss was $2,600 \mathrm{~mL}$ and the volume of autohemotransfusion was $1,250 \mathrm{~mL}$. The RHV was occluded for 30 minutes during the outflow reconstruction of $S_{5}$ and $S_{6}$. The patient recovered uneventfully except for a transient elevation of total bilirubin, and was discharged on the $10^{\text {th }}$ postoperative day. A CT scan 29 days after the operation showed significant liver regeneration. Follow-up demonstrated that the patient enjoyed high quality of life and gave birth to a healthy child 2 years after the operation.

\section{Perspectives of Precision Liver Surgery}

The delicacy in the structure and the complexity in function of the liver along with variability in the pathophysiology of liver disease pose exceptional surgical challenges. ${ }^{100}$ Despite the advances in surgical practice involving the liver, it remains impossible to completely rule out uncertainty and acquire total security from error. Though the end state of absolute determinacy might never be realized, the need for artistry generated by uncertainty makes surgery an extraordinarily appealing field and motivated medical development. This interplay will likely never end, with the evolution of medicine a process of diminishing uncertainty.

Although the theoretical and technological foundation of precision liver surgery has been established, the wide reproducibility of this approach to practice is yet to come. To achieve determinacy, disease-related, patient-specific information must be obtained with greater certainty. The goal of personalized medicine would integrate the analysis of disease relevant molecular data to enable us to refine and precisely tailor the surgical service.

Developments in translation research will facilitate the evolution of new rules in liver surgery. These include better prediction of biological behaviors of malignancies, the capacity of the liver to regenerate, management of $I / R$ injury, and exploration of the therapeutic potential of stem cells in liver disease. In clinical practice, evidence-based rules must also be clearly established along with the interpretation of causeeffect relationships between predictive factors and outcomes; this will endow surgical practice with greater predictability and better standardization.

Technologically, digitized medicine has been playing a key role in improving the predictability and controllability of surgical practice. In the near future, a series of novel technologies should be investigated intensively, including 3D quantitative regional assessment of liver function, intraoperative real-time imaging and navigation, as well as systematic optimization of operative techniques, and digital-assisted decision-making equipment.

The paradigm of precision surgery is consistent with the evolution toward a multidisciplinary architecture within health care organizations. This leads to an in-depth patientcentered integration of diagnostic, predictive, and therapeutic technology to functionalize the multidisciplinary team. 
We expect that this approach, precision liver surgery, that incorporates life sciences, information technology, biomedical engineering, and digital imaging technology will ignite a revolution of technology in our field.

Born in the 19th century and having prospered in the 20th, liver surgery is now about to be revolutionized by the idea of precision as we work toward perfecting surgery in the $21^{\text {st }}$ century. The myth of Prometheus is now undergoing realization in a modern setting.

\section{Acknowledgments}

The authors are grateful to Professor Henri Bismuth, Jean Emond, and Henry Pitt for their professional review and inspiring advice. Same thanks to Professor Michael Allen McNutt and Dr. Zeng Xiaolan for their assistance with the manuscript. This work was supported by the National Key Technology R\&D Program of China (2012BAI06B01), the National High-tech R\&D Program of China (2011AA020103), and the National S\&T Major Project for Infectious Diseases of China (2012ZX10002-017).

\section{References}

1 Gawande A. Two hundred years of surgery. N Engl J Med 2012;366 (18):1716-1723

2 Dong JH. [Remodeling the systematic treatment of hepatocellular carcinoma]. Zhonghua Xiao Hua Wai Ke Za Zhi. 2007;6 (1):3-4

3 Dong JH, Huang ZQ. [Precise liver resection-new concept of liver surgery in 21st century]. Zhonghua Wai Ke Za Zhi 2009;47 (21):1601-1605

4 Dong JH, Yang SZ, Duan WD, et al. [Clinical application of precise liver resection techniques in patients with complicated liver space-occupying lesions]. Zhonghua Wai Ke Za Zhi 2009;47 (21):1610-1615

5 Dong JH, Huang ZQ. [To advocate precision liver surgery and recreate the legend of Prometheus]. Zhonghua Xiao Hua Wai Ke Za Zhi. 2010;9(1):4-5

6 Lang H, Junge A, Sitter H, Klotter HJ. Experimental investigation of intraoperative ultrasound in anatomically precise liver resection. J Invest Surg 1995;8(4):253-261

7 Fan ST. Precise hepatectomy guided by the middle hepatic vein. Hepatobiliary Pancreat Dis Int 2007;6(4):430-434

8 Kuhn TS. The Structure of Scientific Revolution. 2nd ed. Chicago, IL: University of Chicago Press; 1970:VIII-IX

9 Brieger GH. The development of surgery: historical aspects important in the origin and development of modern surgical science. In Sabiston DC, ed. Textbook of Surgery: The Biological Basis of Modern Surgical Practice. 15th ed. Philadelphia, PA: WB Saunders; 1997:1-15

10 Fortner JG, Kim DK, Cubilla A, Turnbull A, Pahnke LD, Shils ME. Regional pancreatectomy: en bloc pancreatic, portal vein and lymph node resection. Ann Surg 1977;186(1): $42-50$

11 al-Hadeedi S, Choi TK, Wong J. Extended hepatectomy for hepatocellular carcinoma. Br J Surg 1990;77(11):1247-1250

12 Nimura Y, Kamiya J, Nagino M, et al. Aggressive surgical treatment of hilar cholangiocarcinoma. J Hepatobiliary Pancreat Surg 1998;5(1):52-61

13 Starzl TE, Iwatsuki S, Van Thiel DH, et al. Evolution of liver transplantation. Hepatology 1982;2(5):614-636
14 Murray JE. Human organ transplantation: background and consequences. Science 1992;256(5062):1411-1416

15 Lobritto S, Kato T, Emond J. Living-donor liver transplantation: current perspective. Semin Liver Dis 2012;32(4):333-340

16 Polychronidis A, Laftsidis P, Bounovas A, Simopoulos C. Twenty years of laparoscopic cholecystectomy: Philippe MouretMarch 17, 1987. JSLS 2008;12(1):109-111

17 Reich H, McGlynn F, DeCaprio J, Budin R. Laparoscopic excision of benign liver lesions. Obstet Gynecol 1991;78(5 Pt 2): 956-958

18 Gagner M, Pomp A. Laparoscopic pylorus-preserving pancreatoduodenectomy. Surg Endosc 1994;8(5):408-410

19 Modlin IM, Kidd M, Lye KD. From the lumen to the laparoscope. Arch Surg 2004;139(10):1110-1126

20 Ji WB, Wang HG, Zhao ZM, Duan WD, Lu F, Dong JH. Roboticassisted laparoscopic anatomic hepatectomy in China: initial experience. Ann Surg 2011;253(2):342-348

21 Garud SS, Willingham FF. Natural orifice transluminal endoscopic surgery. Gastrointest Endosc 2012;76(3):491-495

22 Kohn LT, Corrigan JM, Donaldson MS. To Err Is Human: Building a Safer Health System. Washington, DC: National Academy Press; 2000

23 Evidence-Based Medicine Working Group. Evidence-based medicine. A new approach to teaching the practice of medicine. JAMA 1992;268(17):2420-2425

24 Wente MN, Seiler CM, Uhl W, Büchler MW. Perspectives of evidence-based surgery. Dig Surg 2003;20(4):263-269

25 Engel GL. The need for a new medical model: a challenge for biomedicine. Science 1977;196(4286):129-136

26 Robin ED. Determinism and humanism in modern medicine. JAMA 1978;240(21):2273-2275

27 West AF, West RR. Clinical decision-making: coping with uncertainty. Postgrad Med J 2002;78(920):319-321

28 Lee JM, Yoon JH, Kim KW. Diagnosis of hepatocellular carcinoma: newer radiological tools. Semin Oncol 2012;39(4): 399-409

29 Ward J, Guthrie JA, Scott DJ, et al. Hepatocellular carcinoma in the cirrhotic liver: double-contrast MR imaging for diagnosis. Radiology 2000;216(1):154-162

30 Kruskal JB, Kane RA. Intraoperative US of the liver: techniques and clinical applications. Radiographics 2006;26(4):1067-1084

31 Imamura H, Sano K, Sugawara Y, Kokudo N, Makuuchi M. Assessment of hepatic reserve for indication of hepatic resection: decision tree incorporating indocyanine green test. J Hepatobiliary Pancreat Surg 2005;12(1):16-22

32 Fan ST, Lai EC, Lo CM, Ng IO, Wong J. Hospital mortality of major hepatectomy for hepatocellular carcinoma associated with cirrhosis. Arch Surg 1995;130(2):198-203

33 Clavien PA, Petrowsky H, DeOliveira ML, Graf R. Strategies for safer liver surgery and partial liver transplantation. N Engl J Med 2007;356(15):1545-1559

34 Dong JH, Zheng XS, Chen XP, et al. [Consensus on evaluation of hepatic functional reserve before hepatectomy (2011 edition)]. Zhonghua Xiao Hua Wai Ke Za Zhi. 2011;10(1):20-25

35 Kubota K, Makuuchi M, Kusaka K, et al. Measurement of liver volume and hepatic functional reserve as a guide to decisionmaking in resectional surgery for hepatic tumors. Hepatology 1997;26(5):1176-1181

36 Radtke A, Sotiropoulos GC, Molmenti EP, et al. Computer-assisted surgery planning for complex liver resections: when is it helpful? A single-center experience over an 8-year period. Ann Surg 2010;252(5):876-883

37 Schreckenbach T, Liese J, Bechstein WO, Moench C. Posthepatectomy liver failure. Dig Surg 2012;29(1):79-85

38 van den Broek MA, Olde Damink SW, Dejong CH, et al. Liver failure after partial hepatic resection: definition, pathophysiology, risk factors and treatment. Liver Int 2008;28(6):767-780 
39 van Leeuwen MS, Noordzij J, Hennipman A, Feldberg MA. Planning of liver surgery using three dimensional imaging techniques. Eur J Cancer 1995;31A(7-8):1212-1215

40 Torzilli G, Makuuchi M. Intraoperative ultrasonography in liver cancer. Surg Oncol Clin N Am 2003;12(1):91-103

41 Makuuchi M, Hasegawa H, Yamazaki S. Ultrasonically guided subsegmentectomy. Surg Gynecol Obstet 1985;161(4): 346-350

42 Cai SW, Xie Y, Yang SZ, et al. [Application of lasting methylene blue staining in precise hepatectomy]. Zhonghua Xiao Hua Wai Ke Za Zhi. 2010;9(1):28-30

43 Ishizawa T, Fukushima N, Shibahara J, et al. Real-time identification of liver cancers by using indocyanine green fluorescent imaging. Cancer 2009;115(11):2491-2504

44 Beller S, Hünerbein M, Eulenstein S, Lange T, Schlag PM. Feasibility of navigated resection of liver tumors using multiplanar visualization of intraoperative 3-dimensional ultrasound data. Ann Surg 2007;246(2):288-294

45 Mirnezami R, Mirnezami AH, Chandrakumaran K, et al. Shortand long-term outcomes after laparoscopic and open hepatic resection: systematic review and meta-analysis. HPB (Oxford) 2011;13(5):295-308

46 Nguyen KT, Marsh JW, Tsung A, Steel JJ, Gamblin TC, Geller DA. Comparative benefits of laparoscopic vs open hepatic resection: a critical appraisal. Arch Surg 2011;146(3):348-356

47 Imamura H, Seyama Y, Kokudo N, et al. One thousand fifty-six hepatectomies without mortality in 8 years. Arch Surg 2003;138 (11):1198-1206, discussion 1206

48 Lau WY, Lai EC. Salvage surgery following downstaging of unresectable hepatocellular carcinoma-a strategy to increase resectability. Ann Surg Oncol 2007;14(12):3301-3309

49 Lam VW, Spiro C, Laurence JM, et al. A systematic review of clinical response and survival outcomes of downsizing systemic chemotherapy and rescue liver surgery in patients with initially unresectable colorectal liver metastases. Ann Surg Oncol 2012;19 (4):1292-1301

50 van Lienden KP, van den Esschert JW, de GraafW, et al. Portal vein embolization before liver resection: a systematic review. Cardiovasc Intervent Radiol 2013;36(1):25-34

51 Bismuth H. Revisiting liver anatomy and terminology of hepatectomies. Ann Surg 2013;257(3):383-386

52 Strasberg SM. Nomenclature of hepatic anatomy and resections: a review of the Brisbane 2000 system. J Hepatobiliary Pancreat Surg 2005;12(5):351-355

53 Llovet JM, Brú C, Bruix J. Prognosis of hepatocellular carcinoma: the BCLC staging classification. Semin Liver Dis 1999;19(3): 329-338

54 Rahbari NN, Garden OJ, Padbury R, et al. Posthepatectomy liver failure: a definition and grading by the International Study Group of Liver Surgery (ISGLS). Surgery 2011;149(5):713-724

55 Koch M, Garden OJ, Padbury R, et al. Bile leakage after hepatobiliary and pancreatic surgery: a definition and grading of severity by the International Study Group of Liver Surgery. Surgery 2011;149(5):680-688

56 Rahbari NN, Garden OJ, Padbury R, et al. Post-hepatectomy haemorrhage: a definition and grading by the International Study Group of Liver Surgery (ISGLS). HPB (Oxford) 2011;13(8): 528-535

57 Bruix J, Sherman M; Practice Guidelines Committee, American Association for the Study of Liver Diseases. Management of hepatocellular carcinoma. Hepatology 2005;42(5):1208-1236

58 European Association For The Study Of The Liver; European Organisation for Research and Treatment of Cancer. EASL-EORTC clinical practice guidelines: management of hepatocellular carcinoma. J Hepatol 2012;56(4):908-943

59 Kudo M, Izumi N, Kokudo N, et al; HCC Expert Panel of Japan Society of Hepatology. Management of hepatocellular carcinoma in Japan: Consensus-Based Clinical Practice Guidelines proposed by the Japan Society of Hepatology (JSH) 2010 updated version. Dig Dis 2011;29(3):339-364

60 Ye SL. [Expert consensus on standardization of the management of primary liver cancer]. Zhonghua Gan Zang Bing Za Zhi 2009;17 (6):403-410

61 Marshall JC. Surgical decision-making: integrating evidence, inference, and experience. Surg Clin North Am 2006;86(1): 201-215, xii

62 Dong JH, Yang SZ, Xia HT, et al. Aggressive hepatectomy for the curative treatment of bilobar involvement of type IV-A bile duct cyst. Ann Surg 2013;258(1):122-128

63 Jarnagin WR, Gonen M, Fong Y, et al. Improvement in perioperative outcome after hepatic resection: analysis of 1,803 consecutive cases over the past decade. Ann Surg 2002;236(4):397-406, discussion 406-407

64 Poon RT, Fan ST, Lo CM, et al. Improving perioperative outcome expands the role of hepatectomy in management of benign and malignant hepatobiliary diseases: analysis of 1222 consecutive patients from a prospective database. Ann Surg 2004;240 (4):698-708, discussion 708-710

65 Nathan H, Segev DL, Mayo SC, et al. National trends in surgical procedures for hepatocellular carcinoma: 1998-2008. Cancer 2012;118(7):1838-1844

66 Makuuchi M, Sano K. The surgical approach to HCC: our progress and results in Japan. Liver Transpl 2004;10(2, Suppl 1):S46-S52

67 Wei AC, Tung-Ping Poon R, Fan ST, Wong J. Risk factors for perioperative morbidity and mortality after extended hepatectomy for hepatocellular carcinoma. Br J Surg 2003;90(1):33-41

68 Jarnagin WR, Conlon K, Bodniewicz J, et al. A clinical scoring system predicts the yield of diagnostic laparoscopy in patients with potentially resectable hepatic colorectal metastases. Cancer 2001;91(6):1121-1128

69 Hariharan D, Constantinides V, Kocher HM, Tekkis PP. The role of laparoscopy and laparoscopic ultrasound in the preoperative staging of patients with resectable colorectal liver metastases: a meta-analysis. Am J Surg 2012;204(1):84-92

70 Agrawal S, Belghiti J. Oncologic resection for malignant tumors of the liver. Ann Surg 2011;253(4):656-665

71 Wakai T, Shirai Y, Sakata J, et al. Anatomic resection independently improves long-term survival in patients with T1-T2 hepatocellular carcinoma. Ann Surg Oncol 2007;14(4):1356-1365

72 Eguchi S, Kanematsu T, Arii S, et al; Liver Cancer Study Group of Japan. Comparison of the outcomes between an anatomical subsegmentectomy and a non-anatomical minor hepatectomy for single hepatocellular carcinomas based on a Japanese nationwide survey. Surgery 2008;143(4):469-475

73 Sarpel U, Bonavia AS, Grucela A, Roayaie S, Schwartz ME, Labow DM. Does anatomic versus nonanatomic resection affect recurrence and survival in patients undergoing surgery for colorectal liver metastasis? Ann Surg Oncol 2009;16(2):379-384

74 Are C, Gonen M, Zazzali K, et al. The impact of margins on outcome after hepatic resection for colorectal metastasis. Ann Surg 2007;246(2):295-300

75 Cucchetti A, Ercolani G, Cescon M, et al. Impact of subcentimeter margin on outcome after hepatic resection for colorectal metastases: a meta-regression approach. Surgery 2012;151(5): 691-699

76 Ercolani G, Grazi GL, Ravaioli M, et al. The role of lymphadenectomy for liver tumors: further considerations on the appropriateness of treatment strategy. Ann Surg 2004;239(2): 202-209

77 Sun HC, Zhuang PY, Qin LX, et al. Incidence and prognostic values of lymph node metastasis in operable hepatocellular carcinoma and evaluation of routine complete lymphadenectomy. J Surg Oncol 2007;96(1):37-45

78 Choi SB, Kim KS, Choi JY, et al. The prognosis and survival outcome of intrahepatic cholangiocarcinoma following surgical resection: association of lymph node metastasis and lymph node 
dissection with survival. Ann Surg Oncol 2009;16(11): 3048-3056

79 Nagino M, Nimura Y, Nishio H, et al. Hepatectomy with simultaneous resection of the portal vein and hepatic artery for advanced perihilar cholangiocarcinoma: an audit of 50 consecutive cases. Ann Surg 2010;252(1):115-123

80 Aldrighetti L, Pulitanò C, Catena $\mathrm{M}$, et al. Liver resection with portal vein thrombectomy for hepatocellular carcinoma with vascular invasion. Ann Surg Oncol 2009;16(5):1254

81 Moon DB, Hwang S, Wang HJ, et al. Surgical outcomes of hepatocellular carcinoma with bile duct tumor thrombus: a Korean multicenter study. World J Surg 2013;37(2):443-451

82 Liu CL, Fan ST, Cheung ST, Lo CM, Ng IO, Wong J. Anterior approach versus conventional approach right hepatic resection for large hepatocellular carcinoma: a prospective randomized controlled study. Ann Surg 2006;244(2):194-203

83 Ferrero A, Viganò L, Polastri R, et al. Postoperative liver dysfunction and future remnant liver: where is the limit? Results of a prospective study. World J Surg 2007;31(8):1643-1651

84 Urata K, Kawasaki S, Matsunami H, et al. Calculation of child and adult standard liver volume for liver transplantation. Hepatology 1995;21(5):1317-1321

85 Dong JH. Safety validation of different decision trees in patients with liver tumor using fifty-fifty criteria. Paper presented at: The 10th World Congress of the International Hepato-PancreatoBiliary Association; July, 3, 2012; Paris, France

86 Rocha FG, Matsuo K, Blumgart LH, Jarnagin WR. Hilar cholangiocarcinoma: the Memorial Sloan-Kettering Cancer Center experience. J Hepatobiliary Pancreat Sci 2010;17(4):490-496

87 Patel AA, Torres DM, Harrison SA. Effect of weight loss on nonalcoholic fatty liver disease. J Clin Gastroenterol 2009;43 (10):970-974

88 D'Angelica M, Kornprat P, Gonen M, et al. Lack of evidence for increased operative morbidity after hepatectomy with perioperative use of bevacizumab: a matched case-control study. Ann Surg Oncol 2007;14(2):759-765

89 Schnitzbauer AA, Lang SA, Goessmann H, et al. Right portal vein ligation combined with in situ splitting induces rapid left lateral liver lobe hypertrophy enabling 2-staged extended right hepatic resection in small-for-size settings. Ann Surg 2012;255(3): 405-414

90 Knoefel WT, Gabor I, Rehders A, et al. In situ liver transection with portal vein ligation for rapid growth of the future liver remnant in two-stage liver resection. Br J Surg 2013;100(3):388-394

91 Elias D, Goharin A, El Otmany A, et al. Usefulness of intraoperative radiofrequency thermoablation of liver tumours associated or not with hepatectomy. Eur J Surg Oncol 2000;26(8):763-769

92 Hemming AW, Reed AI, Langham MR Jr, Fujita S, Howard RJ. Combined resection of the liver and inferior vena cava for hepatic malignancy. Ann Surg 2004;239(5):712-719, discussion 719-721

93 Azoulay D, Andreani P, Maggi U, et al. Combined liver resection and reconstruction of the supra-renal vena cava: the Paul Brousse experience. Ann Surg 2006;244(1):80-88
94 Fu SY, Lau WY, Li GG, et al. A prospective randomized controlled trial to compare Pringle maneuver, hemihepatic vascular inflow occlusion, and main portal vein inflow occlusion in partial hepatectomy. Am J Surg 2011;201(1):62-69

95 Chen YW, Li CH, Zhang AQ Yang SZ, Zhang WZ, Dong JH. Preserving hepatic artery flow during portal triad blood inflow occlusion reduces liver ischemia-reperfusion injury in rats. J Surg Res 2012;174(1):150-156

96 Wang PF, Li CH, Chen YW, Zhang AQ, Cai SW, Dong JH. Preserving hepatic artery flow during portal triad blood inflow occlusion improves remnant liver regeneration in rats after partial hepatectomy. J Surg Res 2013;181(2):329-336

97 Brüning A, Mylonas I. Selective occlusion of the portal vein while maintaining hepatic artery blood flow during partial hepatectomy: a new method for preventing ischemia-reperfusion injury? J Surg Res 2012; [Epub ahead of print]

98 Huang ZQ Xu LN, Yang T, et al. Hepatic resection: an analysis of the impact of operative and perioperative factors on morbidity and mortality rates in 2008 consecutive hepatectomy cases. Chin Med J (Engl) 2009;122(19):2268-2277

99 Katz SC, Shia J, Liau KH, et al. Operative blood loss independently predicts recurrence and survival after resection of hepatocellular carcinoma. Ann Surg 2009;249(4):617-623

100 Jones RM, Moulton CE, Hardy KJ. Central venous pressure and its effect on blood loss during liver resection. Br J Surg 1998;85 (8):1058-1060

101 Bismuth H, Castaing D, Garden OJ. Major hepatic resection under total vascular exclusion. Ann Surg 1989;210(1):13-19

102 Azoulay D, Eshkenazy R, Andreani P, et al. In situ hypothermic perfusion of the liver versus standard total vascular exclusion for complex liver resection. Ann Surg 2005;241(2):277-285

103 Raab R, Schlitt HJ, Oldhafer KJ, Bornscheuer A, Lang H, Pichlmayr R. Ex-vivo resection techniques in tissue-preserving surgery for liver malignancies. Langenbecks Arch Surg 2000;385(3):179-184

104 Zhang KM, Hu XW, Dong JH, et al. Ex-situ liver surgery without venovenous bypass. World J Gastroenterol 2012;18(48):7290-7295

105 Buell JF, Cherqui D, Geller DA, et al; World Consensus Conference on Laparoscopic Surgery. The international position on laparoscopic liver surgery: The Louisville Statement, 2008. Ann Surg 2009;250(5):825-830

106 Yohannes P, Rotariu P, Pinto P, Smith AD, Lee BR. Comparison of robotic versus laparoscopic skills: is there a difference in the learning curve? Urology 2002;60(1):39-45, discussion 45

107 Kehlet H, Wilmore DW. Evidence-based surgical care and the evolution of fast-track surgery. Ann Surg 2008;248(2):189-198

108 van Dam RM, Hendry PO, Coolsen MM, et al; Enhanced Recovery After Surgery (ERAS) Group. Initial experience with a multimodal enhanced recovery programme in patients undergoing liver resection. Br J Surg 2008;95(8):969-975

109 Søreide K, Søreide JA. Bile duct cyst as precursor to biliary tract cancer. Ann Surg Oncol 2007;14(3):1200-1211

110 Ulrich F, Pratschke J, Pascher A, et al. Long-term outcome of liver resection and transplantation for Caroli disease and syndrome. Ann Surg 2008;247(2):357-364 